QUIPUKAMAYOC $\mid$ Revista de la Facultad de Ciencias Contables

Vol. 20 N. 38 pp. 50-58 (2012) UNMSM, Lima, Perú

ISSN: 1560-9103 (versión impresa) / ISSN: 1609-8196 (versión electrónica)

\title{
LOS RECIENTES ESTUDIOS DE LA BIOÉTICA Y LA NECESIDAD DE QUE LOS EMPRESARIOS ASUMAN LA RESPONSABILIDAD SOCIAL EMPRESARIAL
}

\author{
RECENT STUDIES OF BIOETHICS AND THE NEED THAT THE BUSINESSMEN
}

TO ASSUME THE CORPORATE SOCIAL RESPONSIBILITY

JULio CÉSAR TRUJILLo MEZA*
Docente Principal de la Facultad de Ciencias Contables
Universidad Nacional Mayor de San Marcos-UNMSM
Lima-Perú
[Recepción: Setiembre de 2012/ Conformidad: Octubre de 2012]

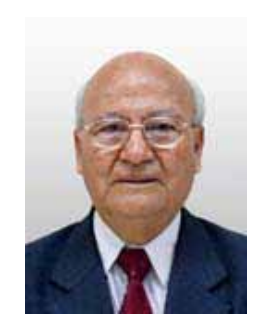

\section{RESUMEN}

El tema considera en lo fundamental un análisis motivacional respecto de los objetivos de la Empresas y de las Instituciones sobre los cuáles deben tenerse en cuenta la necesidad de intensific $r$ la consideración de la Ética y de la Bioética, como criterios y normas de conductas para tener un mejor resultado en la gestión económica y el éxito de la Empresas. Si bien los antecedentes de la aplicación de la bioética tienen un punto de partida por los médicos; también es cierto, que se ha estudiado bastante la aplicación de la ética y de la bioética en el sector empresarial quienes al aplicar sus principios han logrado mejorar su actividad de producción y venta, particularmente ha mejorado todo el entorno con el cual tiene vinculación, siendo reconocida por la sociedad.

Las investigaciones realizadas a la fecha sobre razonamientos bioéticos derivadas a las Empresas, hacen ver que son positivas y valederas en su aplicación conforme puede refle arse en el estudio donde se demuestran índices, dimensiones y criterios positivos para demostrar la necesidad que todas las Empresa y actividades con la inclusión y consideración de los razonamientos bioéticos logran mejorar resultados y la imagen de las empresas realmente muestran un desarrollo en la Responsabilidad Social Empresarial con notable éxito.

Palabras claves: Los Principios de la Bioética y las Empresas Socialmente Responsables.

\section{ABSTRACT}

Fundamentally, the subject matter is a motivational analysis in respect to the objectives of the Companies and of the institutions over which should take into account the need for further consideration of the Ethics and Bioethics, like criteria and standards of behavior for better result in economic management and success of the Companies.

While the backgrounds of the application of bioethics have a starting point in the medicine, it is also true, that has been studied quite the application of ethics and bioethics in the business sector who to apply its principles have improved their production and sales activity. Particularly, they have improved all related environment, being recognized by society.

The researches up to date that has been derived to the companies, about bioethical reasoning, seems be good and valid in their application as be refl cted in the study which show rates, dimensions and positive criteria showing that all companies and activities that include and consider bioethical reasoning, are able to improve their results and the image of the company actually show an improvement in their corporate social responsibility with remarkable success.

I believe that the University should disseminate this knowledge as a new mission applicable to all sectors of the economy to improve and they are considered socially responsible companies and mostly successful.

Keywords: The bioethics principles and socially responsible companies.

\footnotetext{
* Magister en Contabilidad con mención en Tributación. Docente investigador UNMSM. E-mail: estudiotrujillo@yahoo.com
} 


\section{INTRODUCCIÓN}

Los estudios realizados Carroll (1979) y los principios bioéticos propuestos por Beanchamp y Children (1979) dirigidos hacia las Empresas han originado nuevos diseños, con enfoques nuevos en la aplicación ético de los individuos. El mundo globalizado predomínate con el Capitalismo no logra comprender, el cambio y el capitalismo no solo sigue comprando reservas naturales; sino que, que también sigue comprando la vida privada de las personas. Somos una sociedad sin principios ni valores, la subjetividad lo más íntimo de uno se ha vuelto una mercancía, un valor de cambio negociable, siempre obtener el máximo benefi io. Los partidos políticos y algunas Instituciones propician y aceptan a cualquier candidato con tal que fi ancien la campaña electoral, no existe ética todo es por decir, la conciencia reemplazada por el dinero, en suma un capitalismo de alto voltaje.

La Ética que es parte de la filosofía que trata de la moral ${ }^{1}$ y Moral, la apreciación del entendimiento o de la conciencia. Ciencia que trata del bien de las acciones humanas en orden a su bondad o malicia. La Ética como ciencia de las costumbres que se ocupa de lo moral y de las obligaciones del hombre ${ }^{2}$.Son conceptos que tienen que ver con las actitudes o acciones humanas desarrolladas con la bondad o con la malicia y la bioética, como ciencia de principios y razonamientos en el campo ético, si bien es aplicado desde hace mucho tiempo por la medicina y por ello la comprensión, capacidad y desarrollo de la bondad en los médicos es mayor, en nuestro tiempo urge que no solo los médicos deberían conocer aquellos razonamientos; sino que, conociendo la profundidad de la Ética y juntándola con los razonamientos o principios bioéticas, deberían ser considerados por todos los sectores de la actividad económica, Instituciones, Universidades, Gremios y demás.

El día miércoles 19 de Setiembre del 2012, en la página $10^{3}$, el Presidente del Comité de Vigilancia Ética y Deontológica del Colegio Médico del Perú, Dante Añaños, ha propuesto la: "Creación de un Comité Nacional de Bioética para vigilar y concertar sobre la aplicación de los principios de esta Ciencia en las Actividades de Asistencia de Salud y en aquellas no asistenciales".

La propuesta planteada es oportuna y necesaria a los efectos de difundir la Ética y la Bioética para todos los sectores de la actividad Económica, Social, Comercial Institucional de entidades de toda naturaleza si queremos rescatar los principios de orden moral, de actitudes y conductas; sin perjuicio de ello, por la cantidad de trabajadores, Empresarios y el sistema social circundante y vinculante, es conveniente abordar los estudios y la Investigación existentes a nivel de la incidencia que debe tener la ética y la bioética en las Empresas para que las Empresas actúen socialmente responsables.

Es importante mencionar que hace aproximadamente 60 días que salió en un diario local una relación de empresas que habían sido premiadas por su actuación y calificación de "Empresas Socialmente Responsables", en ella figuraban importantes empresas dedicadas a las diversas actividades, inclusive Empresas de Auditoria vinculadas con sus casas matrices del Extranjero. Lamentablemente no había mayor información; pero de dicha aparente fi ción a la realidad objetiva había mucha distancia porque dichas empre-

Diccionario Manual e Ilustrado de la Lengua Española, Pág. 45

Diccionario de Derecho Usual, Guillermo Cabanellas, Pág.132.

Diario La Primera del 19/09/2012, Pág. 10. 
sas antes, ahora y seguro en el futuro seguirán igual en todo caso no se ha estudiado el concepto de ESR.

\section{LAS ACTIVIDADES EMPRESARIALES DE TODA NATURALEZA}

Las Administraciones de las Empresas generalmente están a cargo de Administradores en formación o de otras profesiones por el conocimiento y la práctica, se han visto escándalos empresariales de diferente magnitud. El desarrollo del genoma humano que la tecnología, llevan a plantear ,diversos conflictos éticos impensables en el siglo pasado y en el presente siglo, la ideología neoliberal, el libre mercado, el capitalismo, la tendencia de la privatización de los bienes del Estado hasta servicios de toda naturaleza.

La Bioética, en el campo empresarial comenzó estudiando las implicancias del desarrollo de la ciencia biológica; de otro lado, la ética empresarial ha examinado los principios y problemas éticos relacionados con el mundo de los negocios.

La responsabilidad Social empresarial, es un modelo de gestión empresarial que incorpora las decisiones económicas, sociales, ambientales en sus operaciones en benefi io de los diferentes grupos con los cuales tiene que trabajar, siendo el criterio ético fundamental que regula esta disciplina el respeto al ser humano y su dignidad. La ciencia de la bioética, los principios o los razonamientos como quiera llamarse son los siguientes:

a)La Autonomía.- Cuando la persona tiene un entendimiento adecuado que le permita gobernarse a sí misma, libre de restricciones externas o limitaciones personales que se lo impidan, lo que le permite actuar de acuerdo al plan personal que elija ${ }^{4}$. b) La beneficencia.- Es la obligación de actuar en beneficio de otros promoviendo sus legitimas intereses y suprimiendo perjuicios.

c) La no maleficencia.- Referido al abstenerse de realizar acciones que puedan causar daños o perjuicios a otros. Es un imperativo ético valido no solo e en ámbito biomédico sino en el que muchas reglas básicas de la moral común, como no matar, no causar dolor no romper promesas, no traicionar, etc. ${ }^{5}$ deben ser validas.

d)La Justicia.- Se debe tratar a cada uno como corresponda con la finalidad de disminuir las situaciones de desigualdad (ideológica, social, cultural, económica, etc.).El concepto puede tomarse en lo formal (tratar igual a los iguales y desigual a los desiguales); y de otro lado, en lo material determina las características relevantes para la distribución de los recursos y las cargas.

\section{LOS ANTECEDENTES DE LA BIOÉTICA EN SUS INICIOS}

A los efectos de abordar el tema, necesariamente debemos observar quienes se introdujeron o profundizaron en el tema con criterios científi os.

a)El oncólogo Van Rensselver Potter, utilizó el término por primera vez en 1970, desde aquel entonces muchos estudiosos han aportado nuevas concepciones, métodos y justificaciones (Santos Vargas ,2006).

Algunas reducen a la ética a los temas biológicos, otras lo relacionan con el tratamiento médico y los avances tecnológicos, otros le dan un sentido más amplio para todas aquellas que afecte a los organismos

4 Beancham,2007

5 Ibidem supra 
pero en lo fundamental y de fondo es que lo ético que regula esta disciplina es el respeto al ser humano y a su dignidad

b) Los experimentos médicos fueron inicialmente con los prisioneros en los campos de concentración de la Alemania Nazi y otras investigaciones con procedimientos poco o dudosamente éticos, como las practicas del Hospital de Judíos de Enfermedades Crónicas (Brooklyn, 1963) o la Escuela de Nillowbrook (Nueva York, 1963) y el Estudio de Sífilis de la Universidad de Tuskegee (1972) acciones que fueron calculando la necesidad de regulación, declaraciones a favor de las víctimas de estos experimentos.

Las primeras declaraciones de Bioética surgen de los esfuerzos para regular la investigación y la experimentación médica, tales como el código de Nuremberg (1947), la declaración de Helsinki (1964) y el informe de Belmont (1979).

c)En 1979, los bioétistas T.L Beanchamp y J.F. Childrens ${ }^{6}$, difundieron los cuatro principios de la bioética: Autonomía, no maleficencia, la beneficencia y justicia en su libro "Principles of Bioethical Ethies". Beanchamp (2007) explica que el principio de autonomía tiene sus raíces en la moral liberal y la tradición política en las cuales es importante la libertad y el derecho a elegir de los individuos.

Estos hechos nos llevan a reflexionar sobre la necesidad del conocimiento más profundo de los principios que la originaron, de las investigaciones y procedimientos aplicados para solidariamente ser derivada a las empresas, las Instituciones donde puedan profundizarse sus conceptos y al ser aplicados tengamos los razonamientos éticos y bioéticas de modo que cambiemos las actividades para mejorar y para conseguir una mayor responsabilidad donde actuamos teniendo en cuenta cada uno de los principios bioéticos antes indicados.

\section{LA RESPONSABILIDAD SOCIAL EMPRESARIAL}

La actividad de las Empresas e instituciones es la tradicional de un mero negocio orientado a la maximización del valor de la Empresa y dirigida a satisfacer a los accionistas sobre la rentabilidad (Shareholders). El propósito de los accionistas está en función de las empresas de proporcionar bienes y servicios que lleven al máximo rendimiento de benefi ios a cierto plazo sin reconocer ninguna responsabilidad social con su entorno circundante más allá del rendimiento económico de la Empresa ${ }^{7}$.

a)A fines del año 1970, Carroll (1979) definió la RSE corporativo como los expectativos económicos, legales éticos y discrecionales que la sociedad tiene sobre la organización, lo que implícitamente recoge la responsabilidad de las empresas hacia diversos sectores sociales afines con la teoría de los grupos de interés. Las responsabilidades económicas referidas a la obligación de las empresas de ser productivas y lucrativas para satisfacer las demandas de consumo de la sociedad.

b)Las obligaciones Económicas destacan que la responsabilidad económica tiene que cumplirse dentro del marco jurídico y legal vigente. Las responsabilidades éticas se refieren a aquellas normas, códigos y

6 T.L. Beanchamp y J.F. Childrens, 1979

7 Friedman, 1970 
normas sociales no escritas con la que deben actuar. La dimensión discrecional se refiere a aquellas obligaciones, particularmente filantrópicas que la empresa asume voluntariamente Carroll (1991).

c) Se argumenta estos cuatro componentes que atienden el espectro completo de las obligaciones de los negocios con la sociedad. Este modelo se enfoca en el tipo de obligaciones que las empresas adquieren a diferencia de la teoría de los grupos de interés que se enfoca a identificar las necesidades y reclamos de los grupos implicados.

d)Aupperle y otras (1985) desarrollan un instrumento de respuesta forzada para medir los grados de orientación hacia la Responsabilidad Social Empresarial basada en una mera teoría provista por Carroll $(1979)^{8}$. Los estudios confirman la dimensión económica, segunda de la legal, la ética y la libre discreción ${ }^{9}$ Aupperle y otras en el año 1985, encontraron una relación inversa entra las dimensiones económicas y la ética, implicando que son mutuamente excluyentes

e)López - Palan y Rivera Cruz (2010) ${ }^{10}$, desarrollaron un estudio en base a lo trabajado por Aupperle y otras (1985), pero con distinta metodológica, plantearon un cuestionario con premisas de las cuatro dimensiones (económica, legal, ética y de libre discreción) de las empresas siguiendo el modelo Carroll (1979) y, además, del razonamiento Bioético. Los resultados de los estudios validaron parcialmente las cuatro dimensiones propuestas por Carroll
(1979), pero no según establecidos teóricamente. En todas las dimensiones, excepto en las económicas, estuvieron presente al razonamiento bioética y las consideraciones éticas. La idea debe ser cambiar el criterio tradicional del negocio orientado exclusivamente a la rentabilidad extrema del valor de la empresa.

f)La Responsabilidad empresarial ha generado una bibliografía extensa en los empresarios y en los ambientes académicos y sigue generando y no obstante ello sigue habiendo mucha confusión conceptual y terminológica, haciendo más complejo su entendimiento por la multiplicidad de conceptos y estrategias; sin embargo un gran sector coincide en que el nuevo paradigma de comportamiento de las Empresas y de las grandes comparaciones para que ellas se adapten a estos cambios sociales necesarios surgidos en el marco de la globalización económica ${ }^{11}$.

\section{REALIZACIÓN DE UNA INVESTIGACIÓN DE CARÁCTER INTERNACIONAL CONSIDERANDO LA METODOLOGÍA DE LA INVESTIGACIÓN CIENTÍFICA}

Los Investigadores López - Palan y Rivera Cruz en el año 2012, han decidido continuar con la investigación sobre la aplicación del razonamiento Bioético y la orientación hacia la Responsabilidad Social Empresarial de los Estudiantes de Negocios Latinoamericanos con la participación de estudiantes y docentes universitarios de pre-grado, posgrado y de doctorado de los países de México, Chi-

8 Aupperle y otras , 1985.

9 Aupperle y Summers, 1989; Ibrahim Angeldis 1995; Oncill y otras, 1989, Pinkstm y Carroll 1996, Burton y otros ,2000.

10 López Palan y Rivera Cruz 2010, El razonamiento Bioético y la orientación hacia la responsabilidad Social Empresarial de los Estudiantes de Negocios.

11 Ramiro y Pulido,2009 
le ,El Salvador, Panamá, Puerto Rico y Perú para ello se considero el problema principal y especifi o, las Hipótesis, las variables y los indicadores en el trabajo propuesto.

En el marco teórico conceptual, se analizó la situación en general de las Empresas e Instituciones quienes no aplican los razonamientos de la bioética. Las Empresas en general sólo tiene un referente, la maximización de las utilidades bajo cualquier modalidad o sistema administrativo y conocemos casos de empresas que realizan todos los ajustes posibles solo con el objeto único de la mayor rentabilidad no ven su composición, su entorno, en la sociedad, cuando no cumplan con las normas legales, civiles, laborales, comerciales y tributarias y conllevan fuertes contingencias tributarias.

En las Instituciones, donde el sistema de corrupción es de mayor intensidad observamos que se torna casi común la corrupción bajo cualquier modalidad y todo porque prima el interés, el dinero y los grupos sin considerar los conceptos éticos, mucho menos los razonamientos bioéticos que son necesarios para un cambio en toda la sociedad.

\section{LA INVESTIGACIÓN \\ PROPUESTA SE LLEVO A CABO CON LA PRESENTACIÓN DE MÁS DE QUINIENTAS}

\section{MUESTRAS SIGUIENDO LOS PROCEDIMIENTOS DE LA INVESTIGACIÓN CIENTIFICA.}

Metodología.-Se utilizo el mismo cuestionario que utilizara López- Palan y Rivera Cruz (2010) se ha administrado a estudiantes, docentes del la Escuela de Negocios y otros de cinco países Latinoamericanos.

Se aplicó el análisis factorial para determinar las cuatro dimensiones teóricas de las Empresas y si el razonamiento Bioético es una dimensión relacionada, se determino un índice de orientación hacia la Responsabilidad Social Empresarial y el razonamiento Bioético calculando el promedio de las respuestas a la premisa de la preguntas y a las premisas especificas de los principios bioéticos.

También se determinaron índices de orientación hacia cada una de las dimensiones examinadas y un índice de orientación Bioético Se compararon los promedios de las respuestas mediante pruebas Paired $\mathrm{x}$-Test para identific $r$ diferencias signific tivas y se calculara coefi ientes Chrobach Alpha para determinar la confiabilidad del instrumento.

Además se realizo pruebas Whitney para identific $r$ diferencias signific tivas en las respuestas debido al género y el nivel educativo de los participantes.

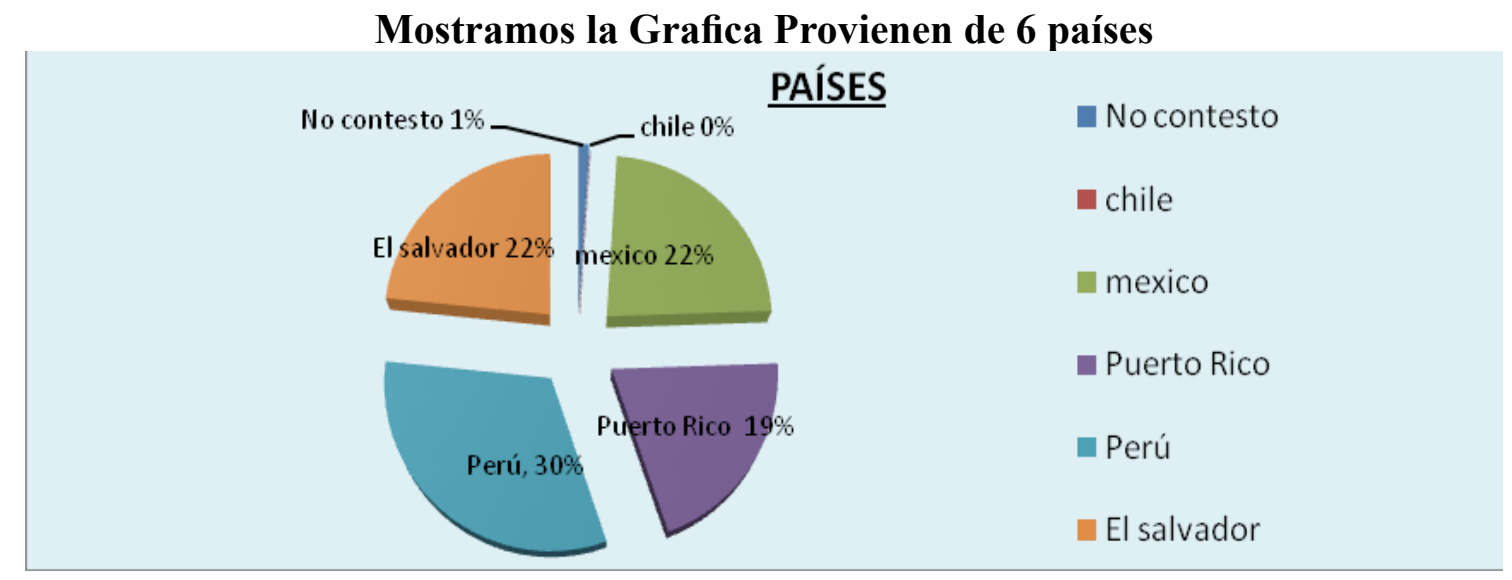


Mostramos la grafica de edades.

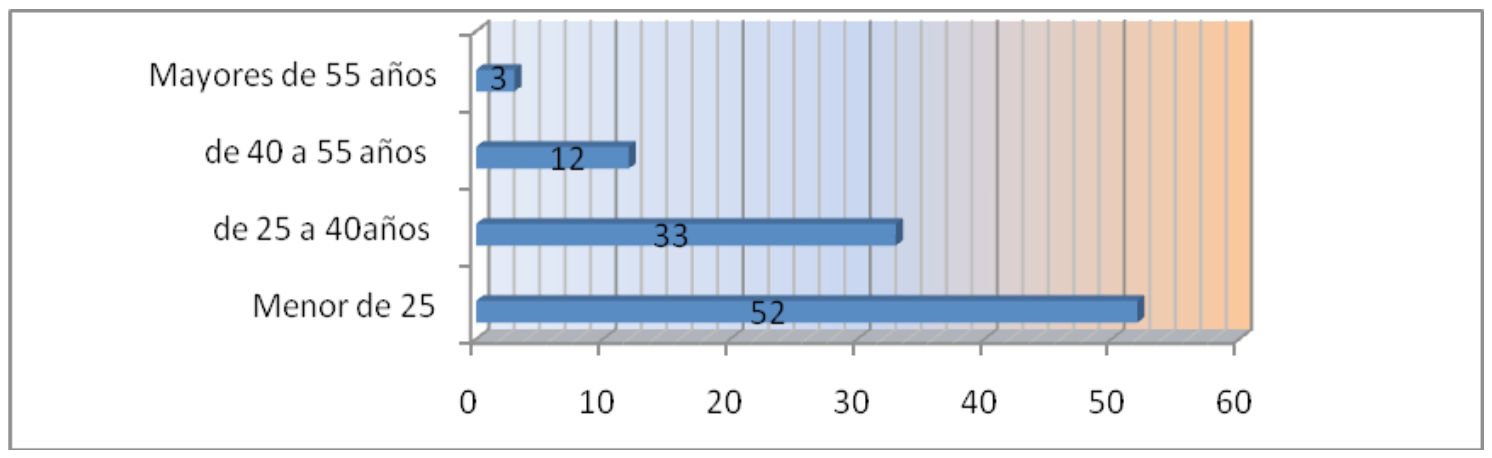

\section{COMPOSICIÓN DE MUESTRA - RESULTADOS}

En el trabajo de la muestra se alcanzó la cantidad de 536 muestras estudiantes latinoamericanos dando como resultado el $48 \%$ hombres; $52 \%$ mujeres. Los encuestados cursan el nivel de Licenciatura 69\%, el nivel de posgrado $29 \%$, más $2 \%$ que no contestaron.

Se observa que el Perú llevo el mayor numero de muestras junto con México y el Salvador.

La grafica $\mathrm{N}^{\circ} 2$ Muestra que el $52 \%$ de los estudiantes son menores de 25 años; muestra que el $33 \%$ están entre los 25 y 40 años; el $12 \%$ tienen edades de 40 a 55 años y el resto del 3\% son mayores de 55 años.

Índice de Orientación hacia la Responsabilidad Empresarial

De la investigación resultan varias tablas sujetas a diferentes análisis, pero en la muestra nos referimos a lo siguiente.
a)La tabla I presenta el promedio obtenido en las premisas que se utilizaron para diferenciar las cuatro dimensiones teóricas da la empresa, según el modelo Carroll (1979) y el razonamiento Bioético.

b) Se presenta los coeficientes Chronbach Alpha de cada una de las dimensiones teóricas.

Los coefi ientes resultaron en valores entre 0,732 y 0,802 . Esto refle a una confiabilidad aceptable para un estudio exploratorio y la mayoría de ellos exceden el límite de 0,70 generalmente aceptado y defendido por Nunally and Berstein (1994) como mínimo deseable.

El coefi iente de la dimensión legal originalmente pero aumenta a 0,802 . Las dimensiones de las Empresas.

\begin{tabular}{|l|c|c|c|}
\hline \multicolumn{1}{|c|}{ PREMISAS } & PROMEDIO & DESVIACIÓN STANDARD & $\begin{array}{c}\text { COEFICIENTE } \\
\text { CHRONBACH } \\
\text { ALPHA }\end{array}$ \\
\hline Dimensión Económica & 84,27752 & 14,711348 & 749 \\
\hline Dimensión Legal & 87,04963 & 14,142688 & 802 \\
\hline Dimensión Ética & 82,95066 & 15,936158 & 732 \\
\hline $\begin{array}{l}\text { Dimensión de Libre } \\
\text { discreción }\end{array}$ & 78,80224, & 15,67512 & 791 \\
\hline $\begin{array}{l}\text { Índice de Razonamiento } \\
\text { Bioético en General }\end{array}$ & 81,3113 & 17,7231425 & 752 \\
\hline $\begin{array}{l}\text { Índice de Promedio de } \\
\text { Orientación RSE }\end{array}$ & 83,044 & 22,138 & 922 \\
\hline
\end{tabular}

56/ QUIPURAMAYoc | Vol. 20(38) 2012 


\section{EL ANÁLISIS FACTORIAL}

Se determinó la adecuasidad del procedimiento mediante las pruebas Kaiser- MyerOlkin y Bartlett Test of Spherincity. Se realizaron una serie de pruebas satisfactorias y los resultados del análisis factorial fue muy efectivo refle ando los promedios y la desviación estándar de cada función, índice y dimensión, llegándose a mostrar correlaciones y coefi ientes de confiabilidad de los índices, tomándose el análisis con los resultados de las Empresas Exitosas y socialmente responsables.

El análisis factorial ha sido aplicado a todas las premisas, funciones y metas, siendo una de las características más signific tivas las aplicadas en las Empresas Exitosas y socialmente Responsables.

\section{CONCLUSIONES}

1. La investigación o estudios logro comprobar los seis objetivos propuestos, quedando en la necesidad de profundizar en sus resultados debidamente analizados.

2. El estudio contribuye con la investigación de la ética y ayuda a la necesidad de aplicar los principios de la ética y bioética para demostrar que los principios bioéticos aplicadas a las Empresas dan como resultado Empresas Socialmente Responsables

3. La investigación comprueba la importancia que tienen las encuestas sobre responsabilidad Social y la forma ética de actuar e invita a seguir con las investigaciones tanto de las Empresas como las Instituciones donde es necesario un cambio en las actitudes y conductas para mejorar los servicios.

4. Es recomendable que en la currícula de estudios se incorpore un curso sobre la responsabilidad Social de la Empresa y dentro de ella la ética y la bioética en su dimensión total para lograr un cambio de los estudiantes y futuros empresarios.

5. La necesidad de hacer talleres de investigación para el manejo y dominio de la Estadística y de los métodos cuantitativos con programas de SPSS y otros aplicados a la investigación en general.

6. Estudiar y analizar la proyección de los principios o del razonamiento de la bioética con el objeto que sea aplicable a todos los campos de las actividades donde pueda ser útil modificar las actitudes $y$ conductas humanas teniendo en cuenta los efectos y la trascendencia de la bioética y lograr con cabal comprensión de la Responsabilidad Social Empresarial.

\section{REFERENCIAS BIBLIOGRÁFICAS}

1. Angeldis, Jhon, Nabil Ibrahim.2004." An Exploratory Study of the Impact of Degree of Business Ethics. Vol.51, Iss.2, p.119 - 128.

2. Arlow, Peter. 1991 "Personal Characteristics in College Students Evaluations of Business Ethics and Corporate Social Responsibility". Journal of Business Ethics. Vol.10, Iss.1, p.63.

3. Aupperle, K.E.: 1991, The Use of Forced - Choice Survey Procedures in Assesssing Corporate Social Orientation, in L.E. Preston (ed.). Research in Corporate Social Performance and Policy, Vol.12, JAI Press ,Greenwinch, CT,pp.266-279.

4. Bay, D.2002. A critical evaluation of the use of the DIT in accounting ethics research. Critical Perspectives o Accounting 13:159-77

5. Beauchamp, Tom L., 2007 .The Four Principles Approach to Health Care 
Ethics in Principles of Health Care Ethics. Second Edition . John Wiley \& Sons.

6. Carroll. A. B.: 1979, "A Three - Dimensional Conceptual Model of Corporate Social Responsibility orientation". Business and Society. Vol.38, Iss. 2, p. 188205.

7. Carroll, A. B.: 1991 Corporate Social Performance Measurement: A Commentary on Methods for Evaluating an Elusive Construct, in L.E. Preston (ed.), Research in Corporate Social Performance and Policy, Vol.12, JAI Press, Greenwich, CT,pp.385-401.

8. Clarkson, M.B.E.1995, "A Stakeholder Framework for Analyzing and Evaluating Corporate Social Performance”. Academy of Management Review, 20, 92117.
9. Freeman, R.E y Velamuri, S.R.:2006, A new approach to CSR: Company stakeholder responsibility, en Kakabadse, A. y Morsing, M., Corporate Social resposability Reconciling aspiration with application,9-23. Palgrave Mcmillan. Gran Bretaña.

10. Friedman , M.:1970, “ The Social Responsibility of Business is to Increase its Profits", New York Times Magazine, 13, 122-126.

11. Ibrahim, Nabil A, Angelidis, John A. 1993." Corporate social responsibility: A comparative analysis of perceptions of top executives and business students". The Mid - Atlantic Journal of Business. Vol .29 ,Iss.3 p. 303 (12pp). 